\title{
LA TRIANGULACIÓN ANALÍTICA COMO RECURSO PARA LA VALIDACIÓN DE ESTUDIOS DE ENCUESTA RECURRENTES E INVESTIGACIONES DE RÉPLICA EN EDUCACIÓN SUPERIOR
}

\section{[Analytical triangulation, a resource for the validity of recurrent survey studies and reply researches in Higher Education]}

por

Article record

$\underline{\text { About authors }}$

HTML format

\author{
Clemente Rodríguez Sabiote (clerosa@ugr.es) \\ Teresa Pozo Llorente (mtpozo@ugr.es) \\ José Gutiérrez Pérez (jguti@ugr.es)
}

$\underline{\text { Ficha del artículo }}$

$\underline{\text { Sobre los autores }}$

Formato HTML

\begin{abstract}
The triangulation understood as technical of confrontation and tool of comparison of different types from analysis of data (analytical triangulation) with a same objective can contribute to validate a survey study and to harness the conclusions that are derived of it. In this article we develop a model of validation of findings by means of analytical triangulation to misalignments observed by two different generations of university students involved in the implantation of a new university curriculum.
\end{abstract}

\section{Keywords}

Validity. Triangulation. Higher Education, Reply Research, Survey Studies.

\section{Resumen}

La triangulación entendida como técnica de confrontación y herramienta de comparación de diferentes tipos de análisis de datos (triangulación analítica) con un mismo objetivo puede contribuir a validar un estudio de encuesta y potenciar las conclusiones que de él se derivan. En este artículo se aplica un modelo de validación de hallazgos mediante triangulación analítica a desajustes observados por dos cohortes diferentes de estudiantes universitarios involucrados en la implantación de un nuevo plan de estudios universitario.

\section{Descriptores}

Validez. Triangulación. Educación Superior, Investigación de Réplica, Estudios de Encuesta.

\section{INTRODUCCIÓN}

Partiendo de las aportaciones de Denzin (1970,1975,1989), Morse (1991), Cowman (1993) o Creswell (1994, 2002), se puede afirmar que la triangulación en el campo de la educación consiste en una estrategia de investigación mediante la cual un mismo objeto de estudio pedagógico es abordado desde diferentes perspectivas de contraste o momentos temporales donde la triangulación se pone en juego al comparar datos; contraponer las perspectivas de diferentes investigadores; o comparar teorías, contextos, ins- 
trumentos, agentes o métodos de forma diacrónica o sincrónica en el tiempo.

Kelle (2001) sugiere que fueron Campbell y Fiske, en 1959, los primeros investigadores en aplicar la triangulación en la investigación social. Cea D’Ancona (1996), por su parte, sitúa el origen de la triangulación en la topografía, la navegación y la logística castrense y destaca también aproximaciones pioneras de la triangulación en la investigación social en los trabajos de Booth (finales del siglo XIX), Vidich y Shapiro (1955); Zelditch (1962); Spradley (1980). Para éste último, el análisis subjetivo de la compleja realidad social está sujeto a la familiarización de los analistas con un "conjunto de manipulaciones, transformaciones, reflexiones y comprobaciones realizadas a partir de los datos con el fin de extraer significado relevante para un problema de investigación” (Spradley, 1980: 70-75).

El desarrollo procedimental de técnicas concretas de triangulación aplicadas a diferentes situaciones y en diferentes momentos históricos ha seguido en cada ámbito disciplinar caminos diferentes según las necesidades de uso, el avance tecnológico y la disponibilidad de una instrumentación más precisa (pensemos en la microscopía, las técnicas de cromatización, los diagnósticos clínicos basados en instrumentación infográfica, electromagnética, radiológica, bioquímica,... ), el desarrollo de técnicas de medición apropiadas y más precisas o el expreso reconocimiento de su valía como acción legitimada social, cultural o científicamente. Su ajuste y acomodación metodológica como procedimiento contrastado, de reconocido prestigio y uso estandarizado en cada comunidad de práctica ha sido diferente a lo largo del tiempo, no cabe duda, según se trate del campo de la ingeniería, la medicina, las ciencias jurídicas, las finanzas, el campo de lo moral, la historiografía, la geografía o el diagnóstico clínico.
El desarrollo de procedimientos autóctonos en cada campo disciplinar está aún lejos de ser tipificado desde una estructura lineal y estándar que marque tendencias y destaque patrones, pues existen tantas modalidades de triangulación como desarrollos y necesidades de uso haya reconocido el ser humano en la historia de su existencia en los diferentes ámbitos gremiales, prácticas profesionales, tecnológicas y científicas. Blaikie (1991:131) argumenta que el término "triangulación significa cosas diferentes para diferentes investigadores"

Así, por ejemplo, en el campo de la investigación criminológica y judicial las técnicas de triangulación son procedimientos de contraste de testimonios y pruebas que contribuyen a reconstruir los hechos, clarificar los motivos de los delitos e imputar responsabilidades a la luz de las evidencias y declaraciones. Triangular para un topógrafo es señalar distintos puntos en el terreno considerándolos como vértices de un triángulo para poder fijar en el plano la posición relativa de esos puntos y sus distancias exactas. En el campo del peritaje arquitectónico, de las valoraciones de siniestralidad o las auditorias empresariales, la triangulación constituye un instrumento esencial de objetivación de evidencias consistentes que permiten poner precio y asignar valor a un terreno o inmueble; delimitar grados de responsabilidad en un accidente en aras de estimar daños, valorar pérdidas y fijar la cuantía de las indemnizaciones; o bien establecer el estado de salud financiero de una determinada organización empresarial objeto de auditoria. En el campo de la medicina y del diagnóstico psicoeducativo la triangulación es un procedimiento que da garantías de que los indicios que se derivan de los análisis clínicos o las pruebas psicométricas apuntan todos hacia una misma dirección interpretativa que ha de orientar las intervenciones y tratamientos.

Los intentos de generar principios de procedimientos amplios en el campo de lo social y cultural no son nuevos. A comienzos del 
siglo pasado desde los ámbitos de la Antropología Social se formularon propuestas de este tipo bajo una denominación común de Método Comparativo. Se suele admitir que Spencer, junto a Taylor y Malinowski iniciaron con distintos matices el método comparativo, consistente en la "acumulación de costumbres e ideas recogidas en diferentes lugares y procedente de períodos muy distintos, a fin de justificar esquemas de desarrollo a los que se había llegado por una vía más especulativa” (Mair, 1978). Las propuestas de Levi-Strauss (1975) con sus teorías sobre el estructuralismo suponen un paso significativo en el intento de dotar de cientificidad a los estudios antropológicos. Su aportación principal es la "comparación estructural”, con variantes en otros autores: "comparación controlada” (Eggan, 1975) o la "comparación constante” (Glasser y Strauss, 1967).

El método comparativo en sus diferentes modalidades se ha transferido al campo educativo con aportaciones centradas en la construcción de teoría local fundamentada, entre las que cabe destacar las aportaciones de Glasser y Strauss (1967), Ragin (1987), así como Ragin y Becker (1992). El método comparado llega, incluso, a generar una rama propia en el seno de las Ciencias de la Educación denominada Educación Comparada.

En el campo educativo, es Denzin (1970) el primero en teorizar sobre triangulación en aspectos tales como conceptualización, tipologías, rasgos y limitaciones y al cual, por ende, debemos la primera clasificación al uso sobre esta técnica de validación de hallazgos mediante contraste y confrontación.

En la investigación educativa, psicológica y social, por su parte, la triangulación es el procedimiento que permite aceptar como razonables las explicaciones sobre el comportamiento y evaluación de las personas y el funcionamiento de los grupos e instituciones.

\section{PROCEDIMIENTOS DE TRIAN- GULACIÓN COMO RECURSOS DE CALIDAD DE LA INVESTIGACIÓN EDUCATIVA}

En este contexto de legitimación de procedimientos de validación ante pares, fuentes, instrumentos, momentos, agentes, métodos,... el cisma entre las aproximaciones cuantitativa y cualitativa ha sido preconizado por no pocas corrientes y autores a lo largo de las tres últimas décadas en base a la búsqueda de criterios de calidad que garanticen la veracidad y legitimidad de los hallazgos de investigación. Los procedimientos de triangulación en el campo de la investigación en Ciencias Sociales han contribuido a superar la fractura paradigmática o "guerra de paradigmas" (Feuer, Towne \& Shavelson, 2002: 7) al ser asumido por la comunidad de investigadores que un rango de métodos pueden emplearse legítimamente para cada tipo de preguntas y que la elección debe estar gobernada por el propósito y las circunstancias de la investigación. Se asume así, que es posible describir el mundo físico y social científicamente, de tal manera que, por ejemplo, múltiples observadores pueden estar de acuerdo sobre lo que ellos ven. Se acepta que "la investigación cualitativa, el estudio de casos y la etnografía pueden hacer contribuciones valiosas a la investigación educativa y que las evidencias que aportan los métodos cualitativos caen dentro del rango de los métodos que pueden considerarse científicos" (Erickson \& Gutiérrez, 2002:21). La ciencia social, y la educativa en particular, como formas del conocimiento humano operan mediante el establecimiento de categorías binarios, clasifican la realidad hasta reducirla a códigos que a la vez que muestran y representan a la misma son capaces de limitarla y costreñirla hasta límites insospechados (Bericat, 1998:11). En este contexto bicefálico o dicotómico habría que asumir dos tipos de consecuencias diferenciadas: a) no cabe mediación entre las prácticas puras que han sido discernidas $\mathrm{y}$, por 
tanto, se ha de actuar optando por alguna de ellas sacrificando indefectiblemente la otra; b) cabe la dualidad y, además, no se sacrifica ninguno de los polos.

De estas dos opciones Ramos (1996:173174) deriva una serie de actuaciones: hacer a la vez lo uno y lo otro (compromiso), hacer lo uno y lo otro en espacios-tiempos sociales diferenciados (compartimentación) y, finalmente, hacer lo uno y lo otro sucesivamente (oscilación).

Mas la interacción no resulta fácil. Los procesos de permeabilización han encontrado sus detractores a lo largo de la historia de la investigación social. Ante el problema de la integración paradigmática se plantean diversas posiciones bien delimitadas. Con Arnal, Del Rincón y Latorre (1994:38) destacamos las siguientes: a) incompatibilidad de paradigmas (Smith y Heshusius, 1986), b) complementariedad de paradigmas (Cook y Reichardt, 1986) у c) unidad epistemológica (Walkers y Evers, 1988).

La primera posición (incompatibilidad de paradigmas) forma parte de lo que Bericat (1998) denomina lógica segregacionista en la que aun aceptándose ambos paradigmas (cuantitativo y cualitativo) no se contempla ningún tipo de fusión entre ellos, sino que su actuación se circunscribe a determinados problemas específicos de cada uno de ellos. Desde este posicionamiento la rigidez entre paradigmas es absoluta. Un mismo objeto de estudio "A" no puede ser abordado por los métodos “ $\mathrm{A}$ ” y " $\mathrm{B}$ ”, sino sólo por el primero. Los mundos cualitativo y cuantitativo existen, pero se obvian y trabajan en solitario.

La segunda y tercera posiciones (complementariedad y unidad epistemológica) integrarían lo que este mismo autor ${ }^{[1]}$ denomina la lógica de integración. En esta posición no sólo se reconoce el mérito de cada método en su respectivo ámbito, sino que, además, se considera posible y fructífera su combinación complementaria para el estudio de muchos fenómenos sociales. La complementa- ción defendida y/o tratada, entre otros, por Cook y Reichardt (1986), Bryman (1988), Jaeger (1988), Goetz y LeCompte (1991), Brannen (1992), Creswell (1994), Erzberger (1998), Flick (1998) o Kelle y Erzberger (1999) podría identificarse con lo que Bericat (1998) llama integración en su versión atemperada. En ella se reconoce que la integración, al menos en algunos casos, es útil y posible. En su versión más radical es posible que toda pregunta o problema de investigación sea abordado mediante un diseño multimétodo que conduciría a resultados más válidos.

Sin ser tan preciso, igualmente, resulta interesante la clasificación que Morgan (1983) elaboró sobre el marco de la actitud convivencial entre paradigmas. Este autor distingue cinco posiciones: supremacía, síntesis, contingencia, dialéctica y todo sirve.

Bericat (1998) desecha las posiciones de la supremacía por reduccionista y la de todo sirve por su evidente ambigüedad y falta de rigor. Retoma, sin embargo, las tres restantes (síntesis, contingencia y dialéctica) para la vía de la integración. De hecho, este autor implementa lo que él denomina la legitimidad científica de la integración materializándola en tres razones/vías para su consecución: vía de la complementación, de la triangulación y de la combinación. Desde este posicionamiento existe complementación cuando un mismo objeto de estudio es abordado mediante dos vías diferentes (cuantitativa y cualitativa) generándose dos productos distintos sobre dicho objeto. Se produce triangulación cuando las orientaciones cuantitativa y cualitativa se utilizan en el reconocimiento de un mismo e idéntico aspecto de la realidad social implementándose los métodos de forma independiente, pero orientados hacia una puesta en común. Finalmente, se produce combinación cuando subsidiariamente un método, sea el cualitativo o el cuantitativo en el otro, con el objeto de fortalecer la validez de este último compensando su propias debilidades. Un desarrollo más 
exhaustivo se puede encontrar en Morgan (1983), Bericat (1998) o Rodríguez (2003).

Por su parte, Massey (1999:184) reconoce que algunos estudios que hacen uso frecuente de la idea de multiplicidad metodológica incurren de forma sistemática en errores conceptuales enmascarados bajo el término triangulación. En la tabla que presentamos a continuación (Tabla1), elaborada por Massey (1999) a partir de un análisis empírico de los diferentes usos metafóricos asignados al término triangulación, resumimos un listado de concepciones erróneas acerca de la llamada triangulación metodológica en la investigación socioeducativa.

Tabla 1. Tipo de errores más comunes en el empleo de la Triangulación Metodológica

\begin{tabular}{l}
\hline $\begin{array}{l}\text { Error Tipo A.- Consiste en usar un segundo método para demostrar la idoneidad del primero como prueba y re- } \\
\text { quisito previo de veracidad. El caso más extendido consiste en el empleo pruebas cuantitativas para validar } \\
\text { hallazgos cualitativos. }\end{array}$ \\
\hline $\begin{array}{l}\text { Error Tipo B.- Reivindica que el acuerdo entre los resultados de dos métodos demuestra la validez del segundo } \\
\text { método tanto como el primero (el principio de mutua confirmación, se conoce también como argumentación cir- } \\
\text { cular). }\end{array}$ \\
\hline Error Tipo C.- Presupone que a cada fenómeno o manifestación social le corresponden idénticos significados. \\
\hline $\begin{array}{l}\text { Error Tipo D.- Asume que el investigador puede llegar a transformar datos cualitativos en cuantitativos y vicever- } \\
\text { sa tanto para escalas como para frases de respuesta a preguntas. }\end{array}$ \\
\hline $\begin{array}{l}\text { Error Tipo E.- Asume que las proposiciones y respuestas obtenidas por diferentes métodos pueden ser convergen- } \\
\text { tes o divergentes, y por tanto, expresarse en términos de acuerdo o desacuerdo. }\end{array}$ \\
\hline Error Tipo F.- Presupone que las fortalezas de un método pueden suplir las debilidades del otro. \\
\hline $\begin{array}{l}\text { Error Tipo G.- Afirmar que los resultados de una investigación corresponden a dos muestras obtenidas de una } \\
\text { misma población sin que la lógica del diseño requiera una argumentación metodológica basada en la probabilidad } \\
\text { y en la estadística . }\end{array}$ \\
\hline
\end{tabular}

\section{APROXIMACIÓN AL CONCEP- TO Y TIPOLOGÍAS BÁSICAS DE LA TRIANGULACIÓN EN LA IN- VESTIGACIÓN EDUCATIVA}

La triangulación es un procedimiento heurístico orientado a documentar y contrastar información según diferentes puntos de vista; de ahí que se pueda hablar de diferentes tipos de triangulación según el foco de contraste: técnicas, agentes, tiempos, métodos, o técnicas de análisis de datos.

Denzin (1970,1978), a propósito de esta idea, contempla una clásica clasificación que se articula en torno a cuatro tipologías básicas.

a) Triangulación de datos.- Dicha triangulación está referida a la confrontación de diferentes fuentes de datos en un estudio. La triangulación se produce cuando existe concordancia o discrepancia entre estas fuentes.
Además, se pueden triangular informantes/personas, tiempos y espacios/contextos.

b) Triangulación teórica.- Puede considerarse como una evaluación de la utilidad y poder de diferentes teorías e hipótesis rivales en una misma investigación. Así pues, consiste en el abordaje de un mismo objeto de estudio desde distintas teorías.

c) Triangulación metodológica.- Referida a la aplicación de diferentes métodos en la misma investigación, ya como idéntico método empleado de forma reiterada en diferentes momentos temporales, ya como diferentes métodos sobre un mismo objeto de estudio.

d) Triangulación de Investigadores.- Dicha triangulación equivale a lo que se conoce como equipos interdisciplinares y consiste en la participación de investigadores de diferentes disciplinas, perspectivas y experiencias en una misma investigación, respetando las distintas aproximaciones que éstos realizan respecto de un problema. 
En este sentido, Janesick (1994:217) añade a las tipologías abordadas una quinta triangulación que denomina triangulación interdisciplinaria (interdisciplinary triangulation), que bien podría identificarse con esta última versión. Cohen y Manion (1994:335) destacan además la triangulación en el tiempo (inspirados en el uso simultáneo de diseños longitudinales y transversales); y la triangulación en el espacio (que superan las limitaciones y barreras culturales de los estudios realizados en un mismo país o subcultura y emplean técnicas cruzadas de contraste cultural).

Arias (2000) también destaca la aparición de otra tipología denominada triangulación en el análisis; aportación ésta que va a resultar crucial para nuestra propuesta de validación por servir de elemento precipitante donde desembocan el resto de tipologías para una comparación final a partir de la cual se puede evaluar el grado de validez de un determinado estudio empírico.

Con Arias (2000), podemos afirmar que la triangulación en el análisis es el uso de dos o más aproximaciones en el análisis de un mismo grupo de datos para propósitos de validación. Ésta se hace comparando resultados de análisis de datos, usando diferentes pruebas estadísticas o diferentes técnicas de análisis cualitativo para evaluar en forma similar los resultados disponibles.

Las posibilidades de triangulación y contraste en una investigación educativa desde la triangulación analítica se han estructurado en los últimos años en las propuestas conocidas como "Mixed Methodology". Este enfoque ha tomado especial relevancia con las aportaciones prágmáticas que según Johnson \& Turner (2003), y Brannen (1992) aporta dicha estrategia, cuyas posibilidades de contraste teórico se resumen en dos opciones:

a) Mixed Models. Mezclando acercamientos cualitativos y cuantitativos simultáneos o paralelos a lo largo de todas las fases de la investigación. Seis modelos mixtos ilustran estas opciones de investigación en lo que respecta a este acercamiento simultáneo, que deja fuera las opciones 1 y 8 por ser acercamientos monometodológicos (Johnson \& Onwuegbuzie (2004:21):

\section{Gráfico 1 - Modelos mixtos}

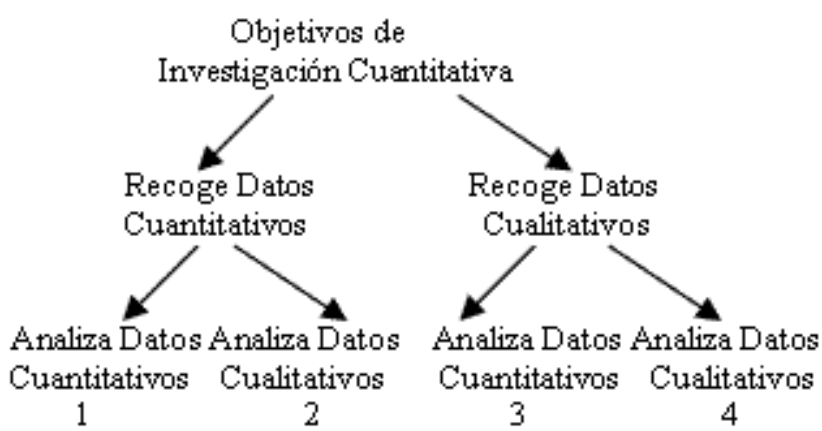

b) Mixed Methods. Desarrollando fases secuenciales de estudios cualitativos y cuantitativos o viceversa a lo largo del tiempo. Nueve diseños metodológicos mixtos posi-

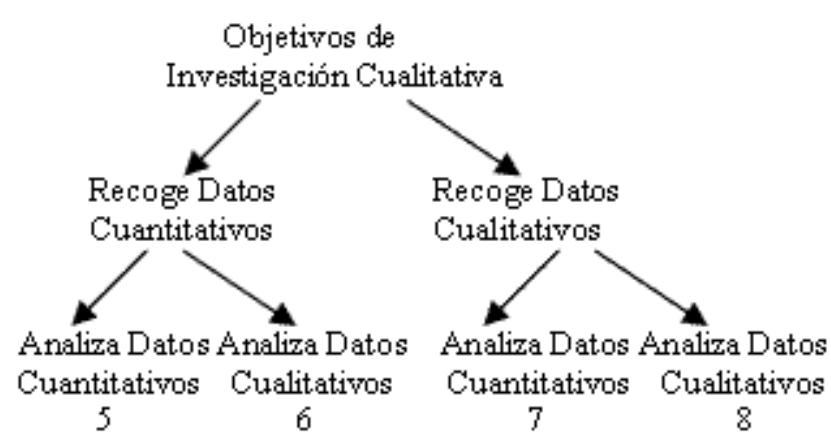

bles de combinación secuencial o concurrente resultan de este enfoque derivado de las propuestas de Morse (1991) y adaptadas por Johnson \& Onwuegbuzie (2004:22): 
Gráfico 2 - Métodos mixtos

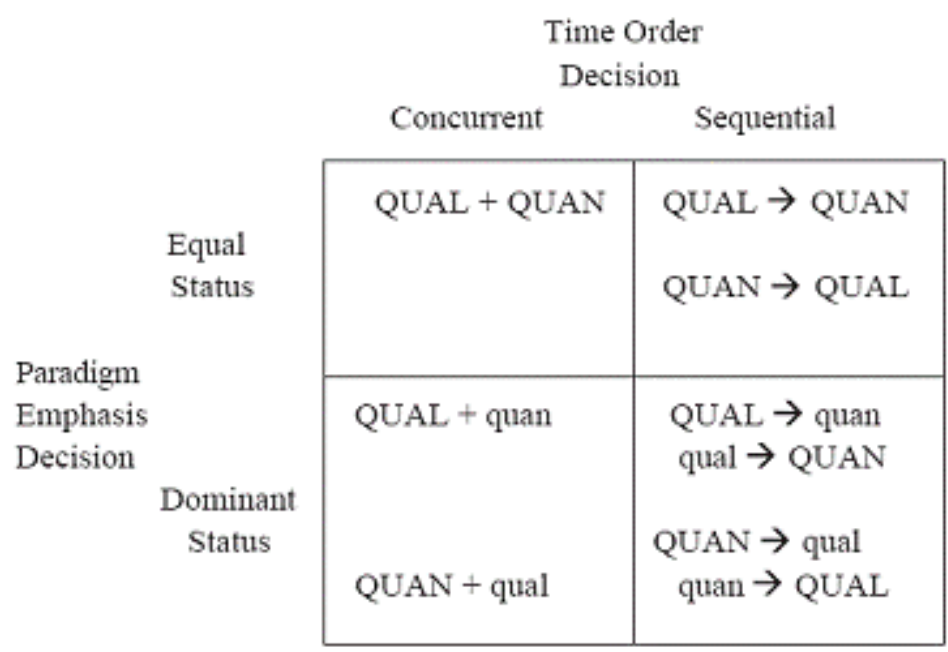

Nota: QUAL = Cualitativa, QUAN = Cuantitativa; la minúscula denota un rango inferior de ese método y la mayúscula prioridad.

El signo “-->” denota secuencia y el signo “+” concurrencia.

\section{MODELO DE TRIANGULACIÓN COMO VALIDACIÓN ANALÍTICA POR FASES APLICADO A UN ES- TUDIO DE ENCUESTA}

El presente trabajo pretende mostrar una propuesta útil de modelización empírica para la determinación del grado de validez de un estudio sobre problemas asociados a la implantación de un plan de estudios universitario. Es un hecho contrastado que la triangulación como estrategia metodológica puede resultar muy valiosa para el establecimiento de validez en los estudios empíricos. Las estrategias de triangulación permiten, por tanto, ejercer un control de calidad sobre los procesos de investigación, ofreciendo pruebas de confianza y garantías de que los resultados y hallazgos que allí se proponen reúnen unos requisitos mínimos de credibilidad, rigor, veracidad y robustez.

En todo caso, es necesario establecer previamente qué fines o metas se quieren lograr con el modelo de triangulación implementado. En este sentido, la mayoría de autores (Erzberger \& Prein, 1997; Erzberger, 1998;
Kelle, 2001; Kelle \& Erzberger, 1999; Kelle \& Erzberger, 2003; Johnstone, 2004) reconocen la existencia de tres modelos de triangulación:

1) Modelo de Triangulación como Validación (The Validity Model)

La triangulación se convierte en la validación mutua de los resultados obtenidos desde diferentes métodos. En este contexto, los resultados cuantitativos y cualitativos pueden y deben converger y llegar a similares conclusiones, razón por la cual se busca la convergencia como punto crucial de decisión. Los resultados divergentes deberían ser interpretados como indicadores de invalidez de una o varias de las metodologías desplegadas.

2) Modelo de Triangulación Complementario (The Complementary Model)

En este modelo de triangulación los resultados cuantitativos y cualitativos pueden relacionar diferentes objetos o fenómenos de estudio y cada uno de ellos complementan al resto. Así pues, la convergencia es vista en el modelo como un aspecto sin valor frente a las divergencias y contradicciones que indican 
que los diferentes métodos relacionan similares aspectos del fenómeno investigado.

3) Modelo de Triangulación Trigonométrico (The Trigonometry Model)

En este modelo la triangulación alcanza su sentido original, o sea, el que tiene en los campos de la navegación, la topografía y logística castrense. De tal forma, que un fenómeno social determinado puede ser caracterizado desde un método "A" a partir de de los métodos "B" y “C”. Asumiendo el primer tipo de triangulación (triangulación como validación) este es el modelo por fases para la validación de estudios empíricos que proponemos siguiendo las recomendaciones de Onwuegbuzie \& Teddilie's (2003:351-383). Dicho modelo consta de 7 fases bien diferenciadas que para nuestro trabajo hemos reducido a 6 al combinar dos de ellas (3 y 4) en la ejemplificación propuesta. Dichas fases son:

Figura 1. Modelo de validación de hallazgos por fases mediante triangulación analítica.

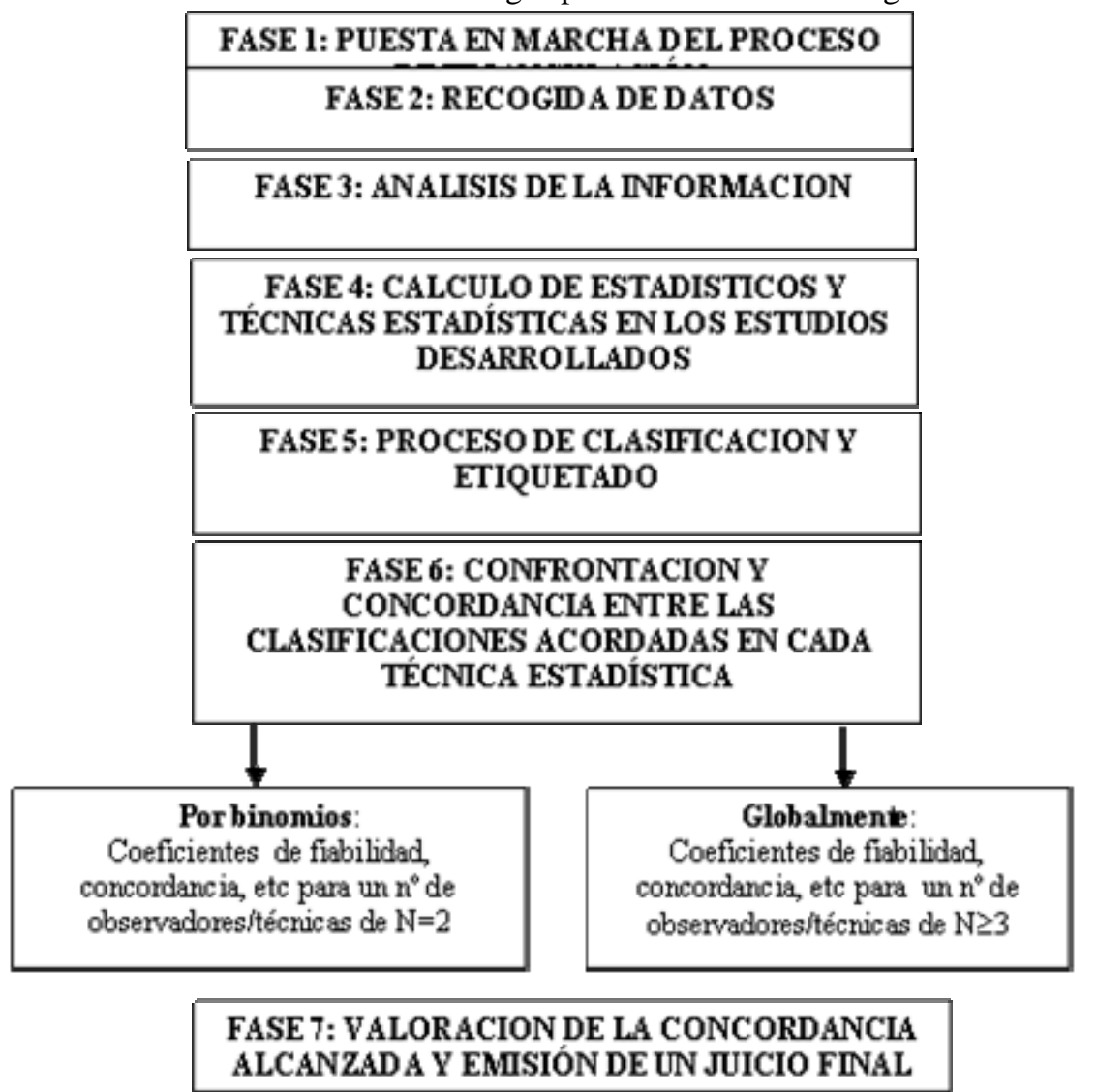

Así pues, tomando como objeto de estudio una investigación de encuesta y el modelo explicitado, estas son las fases desarrolladas:

FASE 1: Puesta en marcha del proceso de triangulación

El estudio implementado en dos cohortes diferentes de estudiantes universitarios que, para este estudio conformaron una sola muestra, consistió en la administración de una escala de estimación de 62 ítems agrupados en 9 metacategorías a 1315 alumnos/as (cohorte 1) y 1263 (cohorte 2) de la Facultad de Ciencias de la Educación de la Universidad de Granada con el objetivo de caracterizar el grado de importancia que dicho alumnado concedía a una colección de desajustes relacionados con la implantación de los nuevos planes de estudio. En cualquier caso, los datos procedentes de ambos años se han analizado como un todo combinado, 
razón por la cual no puede hablarse de triangulación temporal. Sin embargo, la matriz resultante ha sido analizada mediante tres técnicas de análisis diferentes: Medias Aritméticas (MA), Análisis Factorial (AF) y Análisis de Conglomerados (AC). Desde estas coordenadas, con Arias (2000), podemos hablar de triangulación en el análisis. La triangulación en el análisis consiste en el uso de dos o más aproximaciones en el análisis de un mismo grupo de datos para propósi- tos de validación de hallazgos. Ésta se hace comparando resultados de análisis de datos, usando diferentes pruebas estadísticas o diferentes técnicas de análisis cualitativo para evaluar en forma similar los resultados disponibles.

Gráficamente podemos representar nuestra propuesta de triangulación de la siguiente forma:

Figura 2. Esquema del tipo de triangulación desarrollada

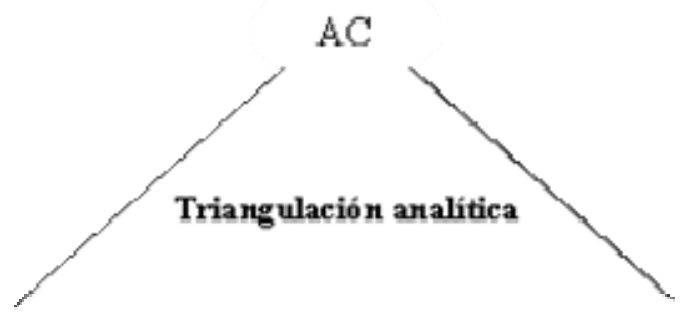

MA

\section{FASE 2: Recogida de datos}

Como hemos señalado en el apartado inmediatamente anterior hemos utilizado como técnica de recogida de información una escala de estimación de 62 ítems con posibles respuestas de valores comprendidos entre 1 : muy en desacuerdo... hasta 5: muy de acuerdo. En cuanto a la muestra y procedimiento de muestreo, dicha escala (denominada PROAC- de problemas académicos-) fue administrada a las dos cohortes a un total de 2578 alumnos/as $(1315+1263)$ de todas las titulaciones de la FCCE de la Universidad de Granada seleccionados mediante muestreo probabilístico estratificado en el que la titu- lación y el curso actuaron de estrato primario y secundario respectivamente.

FASE 3: Análisis de la información recogida y cálculo de estimaciones y técnicas estadísticas

Por la naturaleza de los datos recogidos, claramente cuantitativa, se han implementado diversas técnicas de análisis de carácter descriptivo y multivariante de interdependencia, más exactamente de agrupación y reducción de datos, a saber: Medias aritméticas, Análisis de Conglomerados y Análisis Factorial. Las características de las técnicas multivariantes calculadas son los siguientes:

Tabla 2. Resumen de las principales características de las técnicas de Análisis Multivariante calculadas

\begin{tabular}{|c|c|}
\hline ANÁLISIS DE CONGLOMERADOS & ANÁLISIS FACTORIAL \\
\hline MÉTODO: Conglomerado Jerárquico & $\begin{array}{c}\text { MÉTODO DE EXTRACCIÓN: Componentes } \\
\text { principales }\end{array}$ \\
\hline $\begin{array}{c}\text { MÉTODO DE CONGLOMERACIÓN: Método de } \\
\text { Ward }\end{array}$ & TIPO: Análisis factorial de tipo exploratorio \\
\hline $\begin{array}{c}\text { MEDIDA DE DISIMILITUD EMPLEADA: Euclí- } \\
\text { dea cuadrática }\end{array}$ & MÉTODO DE ROTACIÓN: Varimax \\
\hline & $\begin{array}{c}\text { MÉTODO DE SELECCIÓN DE FACTORES: } \\
\text { Criterio de Kaiser o todo l < } 1 \text { eliminado }\end{array}$ \\
\hline
\end{tabular}


FASE 4: Proceso de clasificación y etique$\underline{\text { tado }}$

Una vez obtenidos los resultados estadísticos de los diferentes niveles (MA, AC y AF) del punto de triangulación contemplado (triangulación en el análisis) los etiquetamos en dos o más categorías en congruencia con el objetivo planteado. En nuestro caso, los criterios de clasificación y las categorías contempladas son los siguientes:

Tabla 3. Criterios de clasificación

\begin{tabular}{|c|}
\hline CRITERIOS DE CLASIFICACIÓN Y ETIQUETADO \\
\hline $\begin{array}{l}\text { - Medias aritméticas obtenidas por las categorías generales de las cohortes } 1 \text { y } \mathbf{2} \text { (MA } \mathbf{M}_{\mathbf{9 7 + 9 8}} \text { ): } \\
\text { Grupo 1. Categorías generales con mayor gravedad aquellas con una media } 33 \\
\text { Grupo 2. Categorías generales con menor gravedad aquellas con una media }<3 \\
\text { - Cálculo de Análisis factorial a las dos matrices de datos de las cohortes } 1 \text { y } 2 \text { conjuntamente (cate- } \\
\text { gorías generales de la escala PROAC) (AF } \mathbf{9 7 + 9 8} \text { ): } \\
\text { Grupo 1. Categorías generales con mayor gravedad aquellas que integran el factor 1: } \\
\text { Grupo 2. Categorías generales de menor gravedad aquellas que constituyen el factor } 2 \\
\text { - Cálculo de Análisis Cluster Jerárquico a las dos matrices de datos de las cohortes } 1 \text { y } 2 \text { conjunta- } \\
\text { mente (categorías generales de la escala PROAC) (AC } \mathbf{9 7 + 9 8} \text { ): } \\
\text { Grupo 1. Categorías de problemas de mayor gravedad las que constituyen el cluster } 2 \\
\text { Grupo 2. Categorías de problemas de menor gravedad las que configuran el cluster } 1 .\end{array}$ \\
\hline
\end{tabular}

Establecidos los criterios de clasificación para las 9 metacategorías contempladas se clasifican en uno de los dos niveles de gravedad propuestos, según las técnicas estadísticas desplegadas. Así obtenemos la siguiente tabla clasificatoria:

Tabla 4. Resultados del proceso de clasificación en el ejemplo propuesto

\begin{tabular}{l|c|c|c}
\hline METACATEGORÍAS & $\mathbf{M A}_{\mathbf{c} 1+\mathbf{c} 2}$ & $\mathbf{A F}_{\mathbf{c} 1+\mathbf{c} 2}$ & $\mathbf{A C}_{\mathbf{c} 1+\mathbf{c} 2}$ \\
\hline PRO- PROFESORADO & 2 & 2 & 2 \\
\hline ECA - ELECCIÓN DE CARRERA & 1 & 1 & 1 \\
\hline GEA - GESTIONES ADMINISTRATIVAS & 2 & 2 & 2 \\
\hline PRA - PRÁCTICAS & 1 & 1 & 1 \\
\hline ALU - ALUMNADO & 2 & 1 & 1 \\
\hline ASI - ASIGNATURA & 2 & 2 & 2 \\
\hline HOR - HORARIO & 2 & 2 & 2 \\
\hline PEA - PERSONAL DE ADMINISTRACIÓN & 1 & 1 & 1 \\
\hline SEI - SERVICIOS E INSTALACIONES & 1 & 1 & 1 \\
\hline
\end{tabular}

FASE 5: Confrontación y concordancia entre las clasificaciones acordadas en cada téc$\underline{\text { nica estadística }}$

A partir de aquí se han calculado diferentes índices de concordancia entre las técnicas de análisis desplegadas. En este sentido, se han contemplado dos diferentes estrategias a la hora de implementar dichos coeficientes. Así, si las comparaciones han sido por bino- mios se han calculado coeficientes Kappa de Cohen (Cohen, 1960), mientras que si se han tomado todas las estrategias de análisis conjuntamente se ha calculado la concordancia canónica (Krippendorf, 1990).

Los resultados de las comparaciones son los que proponemos a continuación. En primer lugar, mostramos los obtenidos por las comparaciones por binomios: 
Tabla 5. Resultados obtenidos por el coeficiente Kappa de Cohen y estadísticos complementarios.

\begin{tabular}{|c|c|c|c|c|}
\hline $\begin{array}{c}\text { Pares de observadores (binomios de técnicas } \\
\text { estadísticas) }\end{array}$ & $\begin{array}{c}\text { Valor } \\
\text { Kappa }\end{array}$ & $\begin{array}{c}\text { Error típ. } \\
\text { asint.* }\end{array}$ & $\begin{array}{c}\text { T aproxi- } \\
\text { mada** }\end{array}$ & $\begin{array}{c}\text { Sig. } \\
\text { aproximada }\end{array}$ \\
\hline MA c1+c2 vs AF c1+c2 & .78 & .20 & 2.40 & $.016^{* * *}$ \\
\hline Ma c1+c2 vs AC c1+c2 & .78 & .20 & 2.40 & $.016^{* * *}$ \\
\hline AF c1+c2 vs AC c1+c2 & 1 & .00 & 3 & $.003^{* * *}$ \\
\hline
\end{tabular}

*Asumiendo la hipótesis alternativa. Medidas simétricas

** Empleando el error típico asintótico basado en la hipótesis nula.

*** Significación estadísticamente significativa asumiendo un alfa bilateral de 0.05.

En segundo lugar, presentamos los resultados obtenidos tomando más de dos estrategias a la vez, es decir, los tres niveles simul- táneamente (técnicas de análisis de datos) teniendo en cuenta que partimos de la siguiente matriz de datos:

Tabla 6. Matriz de partida para el análisis de la Concordancia Canónica de Krippendorf

\begin{tabular}{|c|c|c|c|c|c|c|c|c|c|c|}
\hline & PRO & ECA & GEA & PRA & ALU & ASI & HOR & PEA & SEI & TOTAL \\
\hline $\mathrm{MA}_{\mathrm{c} 1+\mathrm{c} 2}$ & 2 & 1 & 2 & 1 & 2 & 2 & 2 & 1 & 1 & \\
\hline $\mathrm{AF}_{\mathrm{c} 1+\mathrm{c} 2}$ & 2 & 1 & 2 & 1 & 1 & 2 & 2 & 1 & 1 & \\
\hline $\mathrm{AC}_{\mathrm{c} 1+\mathrm{c} 2}$ & 2 & 1 & 2 & 1 & 1 & 2 & 2 & 1 & 1 & \\
\hline $\mathrm{n} 1$ & & 3 & & 3 & 2 & & & 3 & 3 & 14 \\
\hline $\mathrm{n} 2$ & 3 & & 3 & & 1 & 3 & 3 & & & 13 \\
\hline
\end{tabular}

Los datos de dicha matriz han sido sometidos al cálculo de la concordancia canónica en la modalidad propuesta por Krippendorf (1990), es decir:

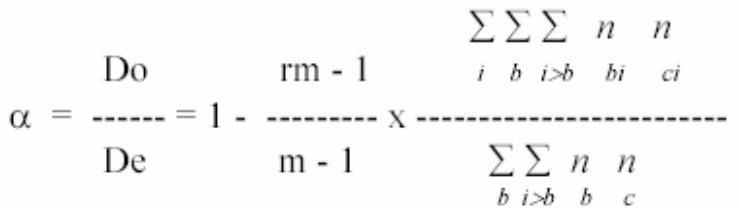

Los resultados del índice a de Krippendorf ascienden a un valor de 0,98 , lo que transformado en porcentaje se convierte en un $98 \%$ de concordancia.

Fase 6: Valoración de la concordancia alcanzada y emisión de un juicio final

Como bien puede apreciarse en una anterior tabla anterior (tabla 4) todos los valores de Kappa de Cohen que pueden considerarse altos. Desde esta perspectiva, podemos afirmar globalmente que la magnitud de la asociación entre los diferentes binomios es importante y, por tanto, a tener en cuenta. Exis- ten numerosos estándares para interpretar los valores del coeficiente Kappa de Cohen (Landis y Koch, 1977; Fleiss, 1981), sin embargo, en lugar de una etiqueta interpretativa, Fleiss, Cohen y Everitt (1969) propusieron estimar el error típico asintótico mediante la ecuación de Fleiss (1981):

$$
\sigma_{Z}=\sqrt{\frac{\mathrm{Pc}+\mathrm{Pc}^{2} \Sigma \mathrm{pi}^{*} \mathrm{pj}^{*}(\mathrm{pi}+\mathrm{pj})}{(1 \mathrm{Pc})^{*} \sqrt{N}}}
$$

y probar la hipótesis nula Ho: $\mathrm{k}=0$ con:

$$
\mathbf{T}=\mathrm{K} / \sigma_{\underline{K}} \cong \mathbf{N}(0,1)
$$


Como puede apreciarse todos los errores típicos asintóticos obtenidos están asociados a valores de “T” altos y estadísticamente significativos $(\mathrm{p} £ 0,05)$, razón por la cual podemos afirmar que los tres valores de Kappa de Cohen obtenidos denotan una considerable concordancia que además de alta es estadísticamente significativa. Por otra parte, el valor de Concordancia Canónica obtenido: 98\% revela, además, la alta concordancia entre las tres técnicas de análisis desplegadas simultáneamente en un esfuerzo común.

\section{CONCLUSIONES}

El despliegue masivo de una colección de estudios (incardinados en una metodología particular) y un conjunto de técnicas (tanto muestrales, de recogida de información, como de análisis) con el fin de caracterizar un objeto común puede considerarse como triangulación múltiple.

La experiencia que mostramos en este trabajo, sin embargo, sólo se centra en un particular tipo de investigación (estudio de encuesta) y singulares tipos de recogida de información (escala de estimación) y muestreo: probabilístico estratificado de tipo proporcional. A cambio propone el análisis de la información recopilada desde diversas fuentes de análisis a fin de poder llegar a puntos de convergencia desde donde poder establecer convincentes argumentos de validación. Guba y Lincoln (1985) denominaron a esto el criterio de “credibilidad" o isomorfismo entre investigación y realidad.

En este contexto, el modelo de validación que proponemos desemboca irremediablemente en el análisis de la información a partir de la cual se modelizan categorías (bajo criterios guiados por la lógica) que finalmente se comparan.

En el estudio que proponemos de ejemplo el fin es caracterizar el grado de importancia que un conjunto de desajustes asociados a la reforma de planes de estudio presentados en escala de estimación tiene para una muestra representativa de alumnos de la Facultad de Ciencias de la Educación de la Universidad de Granada. Para tal fin podrían haberse desplegado las tres técnicas de análisis que proponemos de manera compartimental. Cada una hubiera llegado a conclusiones y hallazgos concretos y hubiera sido tarea del investigador mostrarlos y reflexionar en torno a los mismos en el apartado de discusión. Esta estrategia, que Bericat (1998) denomina de complementación, posee un grado de integración mínimo y su legitimidad se fundamenta en verificar el grado en que cada orientación (o estrategia) es capaz de revelar diferentes e interesantes zonas de la realidad social.

Sin embargo, su comparación simultánea en el análisis y posteriores resultados de semejante información puede resultar muy conveniente para consolidar las conclusiones que de la investigación puedan derivarse. Así pues, además de aumentar el grado de integración, con relación a la anterior propuesta, se pueden captar hechos semejantes de la realidad social desde polos opuestos. Siguiendo, por tanto, la lógica de la integración, en su versión atemperada o moderada, hemos tratado de establecer criterios de etiquetado de las problemáticas (en más graves y menos graves) en cada una de las técnicas desplegadas (medias aritméticas, análisis factorial y análisis de conglomerados o cluster).

Una vez hecho esto, se han clasificado cada una de las categorías objeto de análisis en uno de los dos criterios de etiquetado (más graves/menos graves), tomando como indicador lo indicado por cada técnica de análisis. Finalmente, hemos comparado por binomios, así como globalmente la clasificación que se ha hecho de cada categoría por cada una de las técnicas contempladas con el fin de establecer el grado de concordancia.

En definitiva, la valoración de la concordancia alcanzada, tanto desde la perspectiva comparativa de los binomios, como desde las 
tres técnicas comparadas simultáneamente a la vez, revela el alto grado de simetría o correspondencia alcanzado por las tres técnicas de análisis implementadas (medias aritméticas, análisis de conglomerados y análisis factorial) en el objetivo común de caracterizar el grado de importancia de los desajustes y disfunciones en las dos categorías propuestas: menos graves vs más graves.

En sintonía, pues, con la valoración de los resultados obtenidos (fase 7) y, teniendo en cuenta el tipo de triangulación de partida adoptado: como convergencia, podemos afirmar, por consiguiente, que el estudio de encuesta desplegado cuenta con un alto grado de validez si por ésta entendemos la alta congruencia o correspondencia obtenida entre los diferentes niveles de triangulación contemplados (en nuestro caso las tres técnicas de análisis dispuestas).

Probablemente, además, la más importante contribución de este trabajo consiste en valorar empíricamente la validez de un estudio de encuesta y decidir, en consecuencia, sobre su grado de credibilidad, o lo que es lo mismo, hasta qué punto el aparataje metodológico desplegado (muestreos, instrumentos, análisis de la información...) es capaz de generar un producto final que se aproxime lo máximo posible a la realidad. De todos es sabido que el isomorfismo entre investigación y realidad nunca será acreedor de la perfección. Sin embargo, contar con evidencias empíricas sobre su grado de consecución y valorarlo en consecuencia sí que puede llegar a resultar muy útil.

\section{BIBLIOGRAFÍA}

Arias, M. (2000). Triangulación metodológica: sus principios, alcances y limitaciones. Enfermera, vol. XVIII, (1), 37-57.

Arnal, J., Del Rincón, D. y Latorre, A. (1994). Investigación educativa. Fundamentos y metodología. Barcelona: CEAC.

Bericat, E. (1998). La integración de los métodos cuantitativo y cualitativo en la inves- tigación social. Significado y medida. Barcelona: Ariel.

Blaikie, N. (1991) A critique of the use of triangulation in social research, Quality and Quantity, 25, 115-136

Bolívar Botía, A., Fernández Cruz, M. \& Molina Ruiz, E. (2004, Noviembre). Investigar la identidad profesional del profesorado: Una triangulación secuencial. Forum Qualitative Sozialforschung / Forum: Qualitative Social Research [On-line Journal], 6(1), Art. 12. Disponible en: http://www.qualitative-research.net/fqstexte/1-05/05-1-12-s.htm [Fecha de acceso: octubre/15/2006].

Brannen, J. (Ed.) (1992). Mixing methods: qualitative and quantitative approaches. Aldershot: Avebury.

Bryman, A. (1988). Quantity and quality in social research. London, New York: Routledge.

Cea D’Ancona, M. A. (1996). Metodología Cuantitativa. Estrategias y técnicas de investigación social ( $1^{\mathrm{a}}$ edición). Madrid: Editorial Síntesis.

Chatterji, M. (2005). Evidence on 'What works': an argument for extended-term mixed-method (ETMM) Evaluation Desings. Educational Research 34(5), 1424.

Cohen, J. A. (1960). Coefficient of agreement for nominal scales. Educational and Psychological Measurement, 20, 37-46.

Cohen, L. y Manion, L (1994). Métodos de investigación educativa. Madrid. La Muralla.

Cook, T. D. \& Reichardt, Ch. S. (1986). Métodos cualitativos y cuantitativos en investigación evaluativa. Madrid: Morata.

Cowman S. (1993). Triangulation: a mean of reconciliation in nursing research. Journal of Acvanced Nursing, 18, 788-792.

Cresswell, J. W. (1994). Research design. Qualitative and quantitative approaches. Thousand Oaks: Sage.

Creswell, J.W. (2002). Educational Research: planning, conducting, and evaluating quantitative and qualitative research. New Jersey: Merrill/ Prentice Hall. 
Denzin, N. K. (1970). The research act. Chicago: Aldine Publishing.

Denzin, N. K. (1975). The research act. A theoretical introduction to sociological methods. New York: McGraw Hill.

Denzin N. K. (1989). Strategies of Multiple Triangulation. The Research Act: A theoretical Introduction to Sociological Methods. New York: McGraw Hill.

Eggan, F. (1975). La Antropología Social y el método de la comparación controlada. En Llobera, J. (Comp.). La Antropología como ciencia. Barcelona: Anagrama, 179-202.

Erickson, F. \& Gutiérrez, J.K. (2002). Culture, rigor and science in educational research. Educational Researcher, 31 (8), 2124.

Erzberger, C. (1998). Zahlen und Wörter. Die Verbindung quantitativer und qualitativer Daten undMethoden im Forschungsprozeß. Weinheim: Deutscher Studien Verlag.

Erzberger, C. \& Prein, G. (1997). Triangulation: validity and empirically based hypothesis construction. Quality \& Quantity, 31(2), 141-154.

Feuer, M., Towne, L. \& Shavelson, R.J. (2002). Scientific culture and educational research. Educational Researcher, 31 (8), 414.

Fleiss, J.L. (1981). Statistical methods for rates and proportions. Nueva York: Wiley.

Fleiss, J.L.; Cohen, J. \& Everitt, B.S. (1969). Large-sample standard errors of kappa and weighted kappa. Psychological Bulletin, 72, 295-300.

Flick, U. (1998). An introduction to qualitative research. Thousand Oaks, London, New Delhi: Sage.

Flick, U. (2004). Triangulation. Eine Einführung. Weinheim: Deutscher Studien Verlag.

Glasser, B. G. \& Strauss, A. L. (1967). The discovery of grounded theory: Strategy for qualitative research. Hawthorne, NY: Aldine Publishing Company.

Goetz, J. P. \& LeCompte, M.D. (1991). Qualitative research in social studies education. In J.P. Shaver (Ed.), Handbook of re- search on social studies teaching and learning, a project of the National Council for the Social Studies. New York: MacMillan Publishing Co, 56-67.

Guba, E.G. \& Lincoln, Y.S. (1985). Naturalistic Inquiry. Beverly Hills. Sage.

Gürtler, L. \& Gahleitner, S. B. (2005, September). Conference Report: Fifth Annual Meeting of Qualitative Psychology "Qualitative and Quantitative Approaches to Learning and Instruction" / First Meeting of the Special Interest Group \#17 of the European Association for Research in Learning and Instruction (EARLI) "Mixed Methodology in Psychological Research". Forum Qualitative Sozialforschung / Forum: Qualitative Social Research [On-line Journal], 6(3), Art. 33. Available at: http://www.qualitative-research.net/fqstexte/3-05/05-3-33-e.htm [Date of Access: octubre/15/2006].

Jaeger, R.M. (Ed.) (1988). Complementary methods for research in education. Washington, D.C.: American Educational Research Association.

Janesick, V. (1994). The dance Qualitative Research Design. In Denzin, N.K. \& Lincoln, Y.S. (Eds.), Handbook of qualitative research. London: Sage Publication, 209219.

Johnson, R.B. \& Turner, L.A. (2003). Data collection strategies in mixed methods research. In Abbas Tashakkori \& Charles Teddlie (Dirs.), Handbook of mixed methods in social \& behavioral research. Thousand Oaks: Sage, 297-319.

Johnson, B. \& Onwuegbuzie, A. (2004). Mixed Methods Research: A research paradigm whose time has come. Educational Research 33(7), 14-26.

Johnstone, P. L. (2004). Mixed Methods, Mixed Methodology in Health Services Research. Practice Qualitative Health Research, 14: 259 - 271.

Kelle, U. (2001, February). Sociological Explanations between Micro and Macro and the Integration of Qualitative and Quantitative Methods . Forum Qualitative Sozialforschung / Forum: Qualitative Social Re- 
search, 2(1). Available at: http://qualitativeresearch.net/fqs/fqs-eng.htm [Date of access: March, 02, 2005] .

Kelle, U. \& Erzberger, Ch. (1999). Integration qualitativer und quantitativer Methoden: methodologische Modelle und ihre Bedeutung für die Forschungspraxis. Koelner Zeitschrift fürSoziologie und Sozialpsychologie, 51, 509-531.

Kelle, U. \& Erzberger, Ch. (2003). Making inferences in mixed methods: The rules of integration. In Abbas Tashakkori \& Charles Teddlie (Hrsg.), Handbook of mixed methods in social \& behavioral research. Thousand Oaks: Sage, 457-488.

Landis, J.R. \& Koch, G.G. (1977). An application of hierarchical kappa-type statistics in the assessment of majority agreement among multiple observer. Biometrics, 33, 363-374.

Levi-Strauss, C. (1975). Las tres fuentes de la reflexión etnológica. En J. Llobera, (Comp.): La Antropología como ciencia. Barcelona: Anagrama, 15-24.

Mair, L. (1978). Introducción a la Antropología Social. Madrid: Alianza.

Massey, A. (1999). Methodological triangulation, or how to get lost without being found out. En Massey, A. \& Walford, G. (Eds.). Explorations in methodology, Studies in Educational Ethnography (vol.2, pp.183-197). Stamford: JAI Press. Disponible

http://www.freeyourvoice.co.uk/htm/triangu lation1.htm [junio,07, 2004].

Morgan, G. (Ed.) (1983). Beyond method: strategies for social research. Beverly Hills: Sage.

Morse, J.M. (1991). Approaches to Qualitative-Quantitative Methodological Triangulation. Methodology Corner. Rev. Nursing Research, 40(1), 23-45.

Onwuegbuzie, A.J. \& Leech N. L. (2005). On Becoming a Pragmatic Researcher: The Importance of Combining Quantitative and Qualitative Research Methodologies. International Journal of Social Research Methodology, Vol. 8(5), 375 - 387.
Onwuegbuzie, A. J. \& Teddilie’s, C. (2003). A framework for analyzing data in mixed methods research. In In Abbas Tashakkori \& Charles Teddlie (Dirs.), Handbook of mixed methods in social \& behavioral research. Thousand Oaks: Sage, 351-383.

Ragin, C. (1987).The comparative method: moving beyond qualitative and quantitative strategies. Berkeley: University of California Press.

Ragin, C. \& Becker, H. (1992). What is a case? Exploring the foundations of social inquiry. New York: Cambridge University Press.

Ramos, J. (1996). Jano y el ornitorrinco: aspectos de la complejidad social. En A. Pérez \& I. Sánchez, (Eds.). Complejidad y Teoría Social. Madrid: CIS, 23-52.

Rodríguez, C. (2003). Métodos y estrategias de integración de los paradigmas en investigación educativa. En L. Herrera ; O. Lorenzo; M.C. Mesa \& I. Alemany (Coords.), Intervención psicoeducativa: una perspectiva multidisciplinar. Granada: Grupo Editorial Universitario, 35-52.

Smith, J.K. \& Heshusius, L. (1986). Classing down the conversation: The end of the quantitative-qualitative debate among educational inquirers. Educational Research, 15 (1), 35-45.

Silverman, D. (1993). Interpreting Qualitative Data. London: Sage.

Spradley, J.P. (1980). The ethnographic interview. New York: Holt, Rinehart \& Winston.

Vidich, A.J. \& Shapiro, G. (1955). A comparison of participant observation and survey data. American Sociological Review, 20, 28-33.

Walkers, R. \& Evers, C.W. (1988). The epistemological unity of educational research, In J.P. Keeves (Ed.). Educational research methodology and measurement: An international handbook. Oxford: Pergamon Press, 145-175

Zelditch (1962). Some methodological problems of field studies. American Journal of Sociology, 67, 566-576. 


\section{NOTAS}

[1] Bericat, E. (1998). La integración de los métodos cuantitativo y cualitativo en la in- vestigación social. Significado y medida. Barcelona. Ariel.

\section{ABOUT THE AUTHORS / SOBRE LOS AUTORES}

Clemente Rodríguez Sabiote (clerosa@ugr.es). Doctor en Pedagogía y profesor asociado con labores de docencia e investigación en la Facultad de Ciencias de la Educación de la Universidad de Granada. Sus líneas de investigación son la evaluación de la calidad en educación superior, educación ambiental y los métodos de investigación y el análisis de datos. Su dirección postal es Dpto. de Métodos de Investigación y Diagnóstico en Educación. Facultad de Ciencias de la Educación. Campus de Cartuja s/n 18071 (Granada).

Teresa Pozo Llorente (mtpozo@ugr.es). Profesora asociada y doctora en Ciencias de la Educación desde 2001. Imparte docencia sobre Métodos de Investigación en Educación Social y Evaluación de Programas, Centros y Profesores en la Facultad de Educación de la Universidad de Granada. Su línea principal de investigación es el uso de metodologías de investigación cualitativa en el estudio de trayectorias profesionales. Su dirección postal es Dpto. de Métodos de Investigación y Diagnóstico en Educación. Facultad de Ciencias de la Educación. Campus de Cartuja s/n 18071 (Granada).

José Gutiérrez Pérez (jguti@ugr.es). José Gutiérrez Pérez es Profesor Titular de Métodos de Investigación y Diagnóstico en Educación en la Universidad de Granada, Dpto, MIDE. Director del Secretariado de Evaluación y Calidad en el Vicerrectorado de Planificación, Calidad y Evaluación. Su línea de investigación principal es la evaluación de programas y organizaciones. Su dirección postal es Dpto. de Métodos de Investigación y Diagnóstico en Educación. Facultad de Ciencias de la Educación. Campus de Cartuja s/n 18071 (Granada). 
Rodríguez Sabiote, C., Pozo Llorente, T., Gutiérrez Pérez, J. (2006). La triangulación analítica como recurso para la validación de estudios de encuesta recurrentes e investigaciones de réplica en Educación Superior.

RELIEVE, v. 12, n. 2, p. 289-305. http://www.uv.es/RELIEVE/v12n2/RELIEVEv12n2_6.htm

\title{
ARTICLE RECORD / FICHA DEL ARTÍCULO
}

\begin{tabular}{|c|c|}
\hline $\begin{array}{l}\text { Reference / } \\
\text { Referencia }\end{array}$ & $\begin{array}{l}\text { Rodríguez Sabiote, Clemente, Pozo Llorente, Teresa, Gutiérrez Pérez, José (2006). La triangulación } \\
\text { analítica como recurso para la validación de estudios de encuesta recurrentes e investigaciones de réplica } \\
\text { en Educación Superior. RELIEVE, v. 12, n. } 2 \text {. } \\
\text { http://www.uv.es/RELIEVE/v12n2/RELIEVEv12n2_6.htm. Consultado en (poner fecha). }\end{array}$ \\
\hline $\begin{array}{l}\text { Title / Títu- } \\
\text { lo }\end{array}$ & $\begin{array}{l}\text { La triangulación analítica como recurso para la validación de estudios de encuesta recurrentes e investi- } \\
\text { gaciones de réplica en Educación Superior. [Analytical triangulation, a resource for the validity of re- } \\
\text { current survey studies and reply researches in Higher Education] }\end{array}$ \\
\hline $\begin{array}{l}\text { Authors / } \\
\text { Autores }\end{array}$ & Clemente Rodríguez Sabiote, Teresa Pozo Llorente, José Gutiérrez Pérez \\
\hline $\begin{array}{l}\text { Review / } \\
\text { Revista }\end{array}$ & Revista ELectrónica de Investigación y EValuación Educativa (RELIEVE), v. 12, n. 2 \\
\hline ISSN & $1134-4032$ \\
\hline $\begin{array}{l}\text { Publication } \\
\text { date / } \\
\text { Fecha de } \\
\text { publicación }\end{array}$ & $\begin{array}{l}2006 \text { (Reception Date: } 2005 \text { December 21; Approval Date: } 2006 \text { November 8; Publication Date: } \\
2006 \text { November 16) }\end{array}$ \\
\hline $\begin{array}{l}\text { Abstract / } \\
\text { Resumen }\end{array}$ & $\begin{array}{l}\text { The triangulation understood as technical of confrontation and tool of comparison of different types } \\
\text { from analysis of data (analytical triangulation) with a same objective can contribute to validate a sur- } \\
\text { vey study and to harness the conclusions that are derived of it. In this article we develop a model of } \\
\text { validation of findings by means of analytical triangulation to misalignments observed by two different } \\
\text { generations of university students involved in the implantation of a new university curriculum. } \\
\text { La triangulación entendida como técnica de confrontación y herramienta de comparación de diferentes } \\
\text { tipos de análisis de datos (triangulación analítica) con un mismo objetivo puede contribuir a validar un } \\
\text { estudio de encuesta y potenciar las conclusiones que de él se derivan. En este artículo se aplica un mo- } \\
\text { delo de validación de hallazgos mediante triangulación analítica a desajustes observados por dos cohor- } \\
\text { tes diferentes de estudiantes universitarios involucrados en la implantación de un nuevo plan de estudios } \\
\text { universitario. }\end{array}$ \\
\hline $\begin{array}{l}\text { Keywords } \\
\text { Descriptores }\end{array}$ & $\begin{array}{l}\text { Validity. Triangulation. Higher Education, Reply Research, Survey Studies } \\
\text { Validez. Triangulación. Educación Superior, Investigación de Réplica, Estudios de Encuesta }\end{array}$ \\
\hline $\begin{array}{l}\text { Institution / } \\
\text { Institución }\end{array}$ & Universidad de Granada (España) \\
\hline $\begin{array}{l}\text { Publication } \\
\text { site / } \\
\text { Dirección }\end{array}$ & http://www.uv.es/RELIEVE \\
\hline $\begin{array}{l}\text { Language / } \\
\text { Idioma }\end{array}$ & Spanish (Title, abstract and keywords in English ) \\
\hline
\end{tabular}

\section{Revista ELectrónica de Investigación y EValuación Educativa (RELIEVE)}

\author{
[ ISSN: 1134-4032 ]
}

(C) Copyright, RELIEVE. Reproduction and distribution of this articles it is authorized if the content is no modified and their origin is indicated (RELIEVE Journal, volume, number and electronic address of the document).

(C) Copyright, RELIEVE. Se autoriza la reproducción y distribución de este artículo siempre que no se modifique el contenido y se indique su origen (RELIEVE, volumen, número y dirección electrónica del documento). 